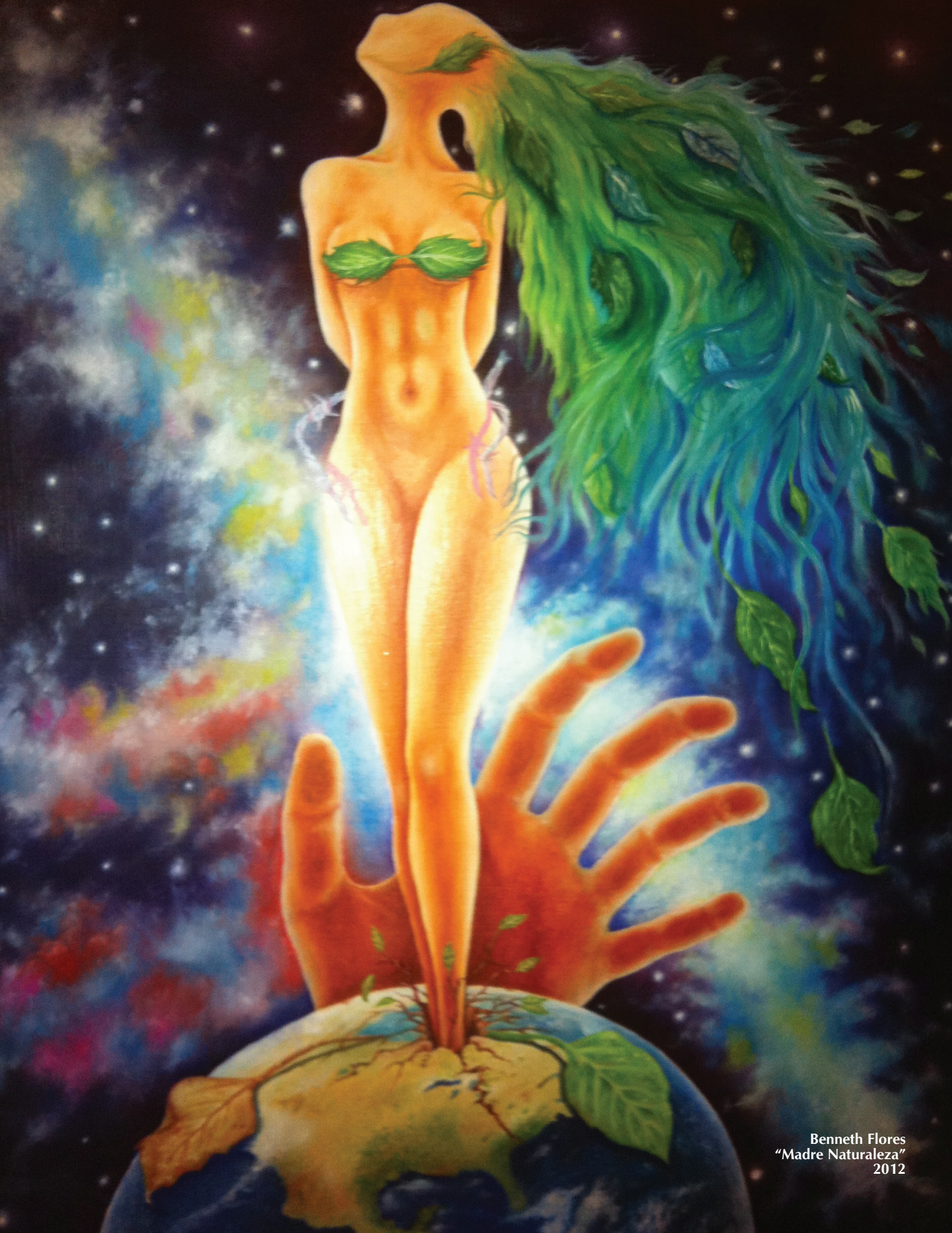




\section{COMENTARIO DE LAS CUMBRES DE LAS NACIONES UNIDAS SOBRE CAMBIO CLIMÁTICO}

Por Abog. Liborio Canales Carias.- Master en Derecho Marítimo y Gestión Portuaria.- Profesor Investigador, Instituto de Investigación Jurídica. Universidad Nacional Autónoma de Honduras. E-mail: abogliborio_canales@yahoo.es

\section{Resumen}

El interés de este artículo es de realizar un breve comentario sobre las Cumbres de Cambio Climático Mundial que se han realizado a lo largo de los años y su preocupación del comportamiento del clima a nivel mundial, en donde cada pais debe de asumir una actitud responsable y tomar conciencia sobre todo los países industrializados, del daño y deterioro de los Recursos Naturales, el Medio Ambiente y la Salud Humana, estableciendo en sus agendas de gobierno los compromisos de reducir el porcentaje de gases de efecto invernadero.

Compartimos que los países logren el desarrollo y crecimiento económico, mediante la utilización racional de los recursos naturales de manera sostenible, manteniendo un equilibrio de los dife-rentes ecosistemas ecológicos, establecidos en los Convenios de las diferentes Cumbres sobre Cambio Climático de las Naciones Unidas.

Palabras Claves: Ambiente, Cumbres, Cambio Climático, Convenciones, Protocolo, países industrializados.

\section{Abstract}

The interest of this paper is to make a brief comment on the Summit of Global Climate Change that have been made over the years and concern the behavior of the global climate, where each country must assume a responsible attitude and become aware of all the industrialized countries, the deterioration and damage of Natural Resour-ces, Environment and Human Health, establishing commitments to reduce the percentage of greenhouse gases to the governmental agenda .

We share that countries achieve economic growth and development through the rational use of natural resources in a sustainable manner, balancing the different ecological systems, established in the agreements of the different Summit on Climate Change of the United Nations.

Keywords: Environment, Summists, climate changes, protocol, industrialize countries. 


\section{INTRODUCCION}

El interés de nuestra investigación "Cumbres de las Naciones Unidas sobre Cambio Climáti-

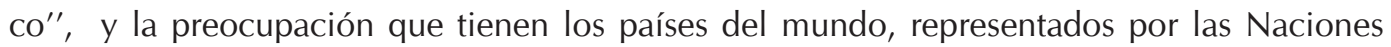
Unidas, nos permite conocer la realidad de cada país sobre el comportamiento del clima a nivel mundial. Los países industrializados como Estados Unidos, China, Japón, India, Rusia, la Unión Europea y otros, son los grandes contaminantes y generan un alto porcentaje de emisión de gases de efecto invernadero.

Esta situación desarrolla diferentes consecuencias como ser: el calentamiento global, inundaciones, sequias, descongelamiento de glaciales, aumento de aguas oceánicas, desertificación, y otros aspectos que afectan el cambio climático mundial.

La importancia de las Cumbres es que de ellas surgen los compromisos y acuerdos que los gobernantes deben cumplir, tomando como base fundamental la "Conferencia Mundial sobre el Clima" (CMC) 1979; prosiguiendo con la primera Conferencia de las Naciones Unidas sobre Cambio Climático y terminando con la aplicación de "Protocolo de Kyoto" aprobado el 11 de diciembre de 1997, donde se busca reducir la emisión de gases y definir estrategias de adaptación, implementado medidas de mitigación que permitan una mejor estabilidad del clima mediante el uso racional de los recursos y el desarrollo sostenible de los mismos.

Es cuando en 1992, la Convención Marco de las Naciones Unidas sobre el Cambio Climático, inicia a considerar de manera cooperativa lo que podían realizar cada uno de los países, para limitar el aumento de la temperatura media global y para hacer frente a los impactos, para entonces, inevitables.

En 1995, los países se dieron cuenta de que las disposiciones de reducción de emisiones en la Convención eran insuficientes y se iniciaron las negociaciones para reforzar la respuesta mundial al cambio climático, dos años más tarde, se adoptó el Protocolo de Kyoto. El Protocolo de Kyoto obligaba jurídicamente a los países desarrollados a los objetivos de reducción de emisiones. El Periodo del primer compromiso del Protocolo comenzó en 2008 y terminó en el 2012, comenzando un segundo compromiso a ser finalizado en el 2016 que aun países desarrollados no han firmado. 


\section{CAPITULO I ANTECEDENTES HISTORICOS}

La Primera Conferencia Mundial sobre el Clima (CMC) se Ileva a cabo en Ginebra en 1979 donde se consideró a nivel internacional el tema de cambio climático como una amenaza real a nivel planetaria. Esta Conferencia adoptó una declaración que exhortaba a los gobiernos a prever y evitar los posibles cambios en el clima provocados por la actividad humana. Al año siguiente se estableció el Programa Mundial sobre el Clima (PMC) con los siguientes propósitos: Establecer un Marco de Cooperación Internacional que permita la investigación y una plataforma que identifique la problemática climática de los años 80 y 90 y evitar el deterioro de la capa de ozono y el calentamiento de la Tierra; utilizar la información climática existente para mejorar la planificación económica, social y ambiental, mejorar la comprensión de los procesos climáticos mediante la investigación y el desarrollo tecnológico. (Alongo, 2010)

La Organización Meteorológica Mundial (OMM) y el Programa de las Naciones Unidas para el Medio Ambiente (PNUMA) crearon el Grupo Intergubernamental de Expertos sobre el Cambio Climático (IPCC) en 1988. La función prioritaria del IPCC; consiste en analizar, de forma exhaustiva y objetiva, la información científica, técnica y socioeconómica para entender los elementos científicos que supone el riesgo del cambio climático provocado por las actividades humanas, sus posibles repercusiones y las posibilidades de adaptación del mismo. El IPCC elabora, asimismo, informes especiales y documentos técnicos sobre temas en los que se consideran necesarios la información y el asesoramiento científicos e independientes, y respalda la Convención Marco de las Naciones Unidas sobre el Cambio Climático (CMCC) mediante su labor sobre las metodologías relativas a los inventarios nacionales de gases de efecto invernadero. (Climatico, 1995)

Mientras que en la Segunda Conferencia Mundial sobre el Clima realizado en Ginebra Suiza a finales de año de 1990, las Naciones Unidas, hizo un Ilamado a todos los países del mundo para iniciar un proceso de negociación que determinara la elaboración de un Tratado Internacional Sobre el Clima. El Comité Intergubernamental de Negociación (CIN), se reunió por primera vez en febrero de 1991 impulsando la iniciativa en relación a la aprobación de una Convención Marco, y más tarde en mayo 9 de 1992, sus representantes gubernamentales adoptaron la Convención Marco de las Naciones Unidas sobre el Cambio Climático, (CMNUCC), este texto de la Convención fue adoptado en la sede de las Naciones Unidas, en Nueva York.

Se abrió a la firma en Río de Janeiro, en la Conferencia sobre Medio Ambiente y Desarrollo, 
conocida como Cumbre de la Tierra, entre el 4 y el 14 de junio de 1992, y posteriormente, en las Naciones Unidas, en Nueva York, desde el 20 de junio de 1992 al 19 de junio de 1993. A esa fecha, la Convención había recibido ya 166 firmas. Luego entró en vigor el 21 de marzo de 1994 y a la fecha tiene 195 Partes (194 Estados y una Organización de Integración Económica).

La Convención tuvo como objetivo último lograr una estabilización de las concentraciones de gases de efecto invernadero en la atmósfera a un nivel que impida perturbaciones peligrosas de carácter antropogénico en el sistema climático. (ONU, 1991)

En virtud de la Convención los países parte se comprometen a:

- $\quad$ Recopilar y compartir la información sobre las emisiones de gases de efecto invernadero, las políticas nacionales y las prácticas adecuadas

- $\quad$ Poner en marcha estrategias nacionales para abordar la problemática de las emisiones de gases de efecto invernadero y adaptarse a los efectos adversos del cambio climático, incluida la provisión de apoyo financiero y tecnológico a los países en desarrollo

- $\quad$ Cooperar para prepararse y adaptarse a los efectos del cambio climático

Desde que entró en vigor, las Partes de la Convención, o sea los países que han ratificado o aceptado el tratado o se han adherido a él, se reúnen anualmente en una Conferencia de las Partes, conocida normalmente por la sigla CP o COP. El objetivo de estas conferencias es impulsar y supervisar la aplicación y continuar las conversaciones sobre la forma más indicada de enfrentar el cambio climático.

Las sucesivas decisiones adoptadas por la Conferencia de las Partes en sus respectivos períodos de sesiones constituyen hoy un conjunto detallado de normas para la aplicación práctica y eficaz de la Convención.

Los órganos que actualmente funcionan en la CMNUCC son:

\section{1.- La Conferencia de las Partes. (COP)}

Es el órgano rector de la convención que como instancia superior de la misma tiene, entre otras responsabilidades, asegurar la plena implementación de la Convención.

\section{2.- Órgano Subsidiario de Asesoramiento Científico y Tecnológico. (OSACT)}

Asesora a la COP sobre los aspectos relacionados al sistema climático, los asuntos ambientales, la tecnología y los métodos. Se reúne dos veces al año.

\section{3.-Órgano Subsidiario de Ejecución.}


Supervisa la aplicación de la Convención, analizando las comunicaciones nacionales presentadas así como los aspectos financieros, incluidos los mecanismos financieros de la Convención, y administrativos. Se reúne dos veces al año.

\section{4.- Organismos vinculados.}

- Fondo para el Medio Ambiente Mundial (FMAM), establecido para facilitar donaciones y canalizar el financiamiento hacia los países en desarrollo y en particular los Países Menos Adelantados y los Estados Insulares en Desarrollo.

- El Panel Intergubernamental sobre el Cambio Climático (IPCC). Desarrolla evaluaciones detallados aproximadamente cada cinco años sobre el estado del conocimiento de la ciencia del cambio climático, los impactos, la vulnerabilidad, la adaptación, la mitigación, los reportes especiales y documentos técnicos.

\section{5.- Observadores.}

Los observadores incluyen a diferentes categorías, entre las que se cuentan los organismos intergubernamentales autorizados a asistir e incluso intervenir, pero no a participar en la toma de decisiones, como por ejemplo el PNUD, PNUMA, UNIDO y otros; las organizaciones no gubernamentales; las instituciones de investigación y académicas; la organizaciones que representan a los actores económicos; los medios de comunicación; los representantes de los pueblos originarios, etc.

\section{6.- Secretariado de la CMNUCC.}

Presta apoyo a la Convención y sus órganos subsidiarios. Se encarga de los preparativos para las reuniones, compila y distribuye estadísticas e información y asiste a los países miembros en el proceso de cumplimiento de los compromisos contraídos en virtud de la Convención. El Secretariado tiene su sede en Bonn, Alemania.

\section{7.- Grupos de expertos.}

Grupo de expertos que asisten a preparar informes, establecer programas de Adaptación y transferencia de tecnología para facilitar la mitigación y la adaptación en los países en desarrollo .

\section{8.- La Junta Ejecutiva.}

Supervisa el Mecanismo de Desarrollo Limpio (MDL) en virtud de lo dispuesto en el Protocolo de Kioto y actúa bajo la autoridad y orientación de la Conferencia de las Partes en calidad de reunión de las Partes en el Protocolo, para la que prepara decisiones que la CMP considera y eventualmente adopta. 


\section{CAPITULO II CUMBRES SOBRE EL CAMBIO CLIMÁTICO}

\section{A.- CUMBRE RIO DE JANERIO (BRASIL)}

En el año de 1992 se realiza la primera Conferencia de las Naciones Unidas sobre Medio Ambiente y Desarrollo, conocida como Cumbre de la Tierra en Rio de Janeiro Brasil, donde se reunieron 178 países y más de 400 representantes de organizaciones no gubernamentales (ONG) los cuales se comprometieron a la firma del Tratado sobre Cambio Climático, y tomar las medidas para la mitigación de la emisión de gases de efecto invernadero. Fue además la primera reunión internacional de magnitud realizada después del final de la Guerra Fría.

En la cumbre de la tierra se consensuó la definición de desarrollo sostenible que años atrás se había presentado en el Informe de Brundlandt. (Desarrollo, 1987) En la Conferencia de Río, la cooperación prevaleció sobre el conflicto; aprobándose varios aspectos que regularían la labor futura como ser: un plan de acción mundial para promover el desarrollo sostenible; la Declaración de Río sobre el Medio Ambiente y el Desarrollo y un conjunto de principios que establecen los derechos civiles y obligaciones de los Estados.

Se abrieron nuevos caminos para el diálogo multilateral, poniendo los intereses globales como su principal preocupación, el significado de la Cumbre de Río fue mucho más allá de los compromisos concretos asumidos, ya que puso de manifiesto las posibilidades de comprensión en un mundo libre de antagonismo ideológico. Los compromisos específicos adoptados por la Conferencia Río-92 incluyen dos Convenciones: una sobre Cambios Climáticos y otra sobre la Biodiversidad, y también una Declaración sobre Florestas. El concepto fundamental de desarrollo sostenible, que combina las aspiraciones compartidas por todos los países al progreso económico y material con la necesidad de una conciencia ecológica.

El principal logro de la Conferencia fue las firmas de los paises a la Convención Marco de las Naciones Unidas sobre el Cambio Climático, que más tarde llevaría al Protocolo de Kioto sobre el cambio climático

\section{B.- CUMBRE DE BERLIN (ALEMANIA)}

Esta cumbre se desarrolló en Alemania en los meses de marzo y abril de 1995. En ésta cumbre, se implementó la Convención de Cambio Climático COP conocida también como COP1, y se realizaron sugerencias para su organización. En esta cumbre las Partes en su primer período de sesiones, examinó los incisos a) y b) del párrafo 2 del artículo 4 de la Convención Marco de las Naciones Unidas sobre el Cambio Climático, y llegó a la conclusión de que esos incisos 
no eran adecuados, se decidió que las obligaciones de los países industrializados no eran las adecuadas por lo que se produce el Mandato de Berlín: Que acuerda poner en ejecución un plan que permita tomar medidas apropiadas para el período posterior al año 2000, en particular el reforzamiento de los compromisos de las Partes mediante la adopción de un protocolo u otro instrumento jurídico.

La ejecución de ese plan se guiará, entre otras cosas, por lo siguiente:

a) "Las Partes deberían proteger el sistema climático en beneficio de las generaciones presentes y futuras, sobre la base de la equidad y de conformidad con sus responsabilidades comunes pero diferenciadas y sus respectivas capacidades.En consecuencia, las Partes que son países desarrollados deberían tomar la iniciativa en lo que respecta a combatir el cambio climático y sus efectos adversos"

\section{C.- CUMBRE DE GINEBRA (SUIZA)}

Ésta convención (COP2) fue abierta en el Palacio de las Naciones, Ginebra, el 8 de julio de 1996 por la Presidenta de la Conferencia en su primer período de sesiones, Sra. Ángela Merkel, Ministra Federal del Medio Ambiente, la Conservación de la Naturaleza y la Seguridad Nuclear de la República Federal de Alemania. Tras dar la bienvenida a todos los participantes en la Conferencia, dijo que ese período de sesiones proporcionaba a la Conferencia de las Partes la oportunidad de realizar una evaluación provisional de la aplicación de la Convención Marco de las Naciones Unidas sobre el Cambio Climático y de los progresos hechos en las negociaciones iniciadas en el primer período de sesiones con la adopción del Mandato de Berlín.

\section{D.- CUMBRE DE KIOTO (JAPON)}

La Cumbre de Kioto, el 11 Diciembre de 1997 fue la III Conferencia de las Partes del Convenio Marco sobre Cambio Climático (COP3), que reunió a 125 paises. El documento elaborado, conocido como Protocolo de Kioto, fue el primer compromiso internacional para frenar el cambio climático que comprometía a los países que lo ratificarán, lograr reducir las emisiones de los gases catalogados como causantes del efecto invernadero. Se le pide a los países industrializados reducir sus emisiones de gases en un 5,2\%, con la finalidad de frenar el calentamiento global.

El Protocolo motivo a los gobiernos a establecer leyes y políticas para cumplir sus compromisos, a las empresas a tener el medio ambiente en cuenta a la hora de tomar decisiones sobre sus inversiones, y además propicio la creación del mercado del carbono. Todos los países industrializados y más de 40 países en desarrollo presentarón oficialmente sus metas 
y medidas de reducción de las emisiones en el marco del Protocolo, pero la respuesta internacional fue insuficiente en un área de crítica importancia. Hasta ese momento la suma total de las promesas oficiales de reducción de las emisiones de todos los países solo asciendía a aproximadamente un $60 \%$ de lo que hacía falta para limitar la subida de la temperatura a 2 grados Celsius, el tope que según los científicos da una probabilidad razonable de evitar los peores impactos del cambio climático. El primer periodo de compromiso del Protocolo de Kyoto concluyo en el 2012.

\section{1.- Mecanismos propuestos por el Protocolo de Kioto para minimizar el impacto económico.}

Muchas de las medidas a tomar para reducir las emisiones de gases de efecto invernadero plantean serios problemas financieros para ciertos sectores, por lo que resulta imprescindible aplicar políticas que reduzcan el inevitable impacto económico.

Por este motivo el protocolo de Kioto incluyó medidas como la de los sumideros de carbono, el cual consiste en aumentar las extensiones forestales y tierras de cultivo que de forma natural absorben importantes cantidades de dióxido de carbono. Se cuenta también con los mecanismos de flexibilidad, que causan controversia para los cuales se toman diferentes tipos de medidas como ser compra venta de emisiones, donde los países adquieren compromisos de reducir la emisión de gases por debajo de los porcentajes establecidos; otra medida es vender esa diferencia a otros países que superan sus límites, de modo que si se reduce el nivel de emisión de gases se obtiene una compensación económica por los países industrializados internacionalmente. Esta situación no debe considerarse como un intercambio comercial, sino más bien como una obligación que tienen los países desarrollados en brindar compensación económica a los países en desarrollo; otras de las medidas es la creación de meca-nismo de Desarrollo Limpio (MDL) que consiste en exportar proyectos de tecnología limpia a países que no han asumido ningún compromiso de reducción, de modo que los exportadores se descuentan la diferencia de emisiones que resulta del abandono de la antigua tecnología y los países menos desarrollados reciben fondos de la implementación conjunta.- Es una medida parecida a la del mecanismo de Desarrollo Limpio, pero con la diferencia de que el intercambio de tecnología se hace entre países con compromiso de emisiones de Gases.

\section{2.- Países Comprometidos en Kioto}

Federación Rusia, Japón, Alemania, Canadá, Reino Unido, Francia, Italia Australia, Ucrania, España, Polonia. 
Países no Comprometidos en Kioto

China, Estados Unidos, India, Corea del Sur, Irán, México, Indonesia, Arabia Saudita, Brasil, Sudáfrica.

\section{E.- CUMBRE DE BUENOS AIRES. (ARGENTINA)}

La cumbre de Buenos Aires, se desarrolló del 02 al 13 de Noviembre de 1998. Fue la IV Conferencia de las partes del Convenio sobre Cambio Climático (COP4). Se adoptó un plan de acción donde se establecían lazos para afinar los detalles sobresalientes de protocolo de Kioto y destacaron temas como los mecanismos financieros que ayudasen a los países en desarrollo a responder a los retos planteados por el Cambio Climático; el desarrollo y la transferencia de tecnología.

Líderes y delegados de más de 180 países del mundo se reunieron para construir un acuerdo en lo que respecta fundamentalmente a crear un nuevo tratado sobre el clima que fije compromisos sobre reducción de emisiones de gases de efecto invernadero que causan el calentamiento del planeta. Llamó la atención que la mayoría de los participantes sostuvieron desde el principio "la importancia de llegar al menos a un acuerdo" hacia el final de la Cumbre. Por ello, los resultados finales arrojados por la Cumbre no satisfacieron todas las expectativas generadas. La falta de consensos en materia de pautas diferenciadas con arreglo a las emisiones nacionales debilitó las posibilidades de alcanzar un acuerdo vinculante entre el conjunto de las partes. Las posiciones encontradas de las principales potencias del planeta cumplieron un rol insoslayable al respecto.

\section{F.- CUMBRE DE BONN. (ALEMANIA)}

La cumbre de Bonn (Alemania) fue realizada el 25 de Octubre y el 5 de Noviembre de 1999, fue la V sesión de la Conferencia de las partes de la Convención Marco de las Naciones Unidas sobre el Cambio Climático (COP5).

La Conferencia de Bonn (Alemania) sirvió para marcar el inicio del protocolo de Kioto puesto que fue firmado por los principales países industrializados (negándose solo los EE.UU, pues afectaría su crecimiento en un $35 \%$ ). Desde este momento el protocolo entro en vigor.

La cumbre de Bonn alcanzo un acuerdo de última hora sobre protocolo de Kioto, Japón, Canadá, Rusia y Australia respaldaron finalmente el acuerdo de compromiso presentado por la Unión Europea. 
El acuerdo de Bonn concluido en Marrakech, contiene la descripción detallada de como tienen que contar los países sus emisiones de efecto invernadero, como serán penalizados si no lo cumplen, como contabilizar el efecto en sus bosques y actividades agrícolas, como se organizaran los proyectos de reducción de contaminación, que ayuda recibirán los países en vías de desarrollo para hacer frente al cambio climático y como hay que informar abiertamente acerca de las actividades de cada uno.

\section{G.- CUMBRE DE LA HAYA. (HOLANDA)}

Fue desarrollada entre el 13 y el 24 de Noviembre del 2000 esta fue VI sesión de Conferencia de las partes de la Convención Marco de las Naciones Unidas sobre el Cambio Climático (COP6). Europa y EE.UU rompieron su diálogo, al subrayar sus discrepancias sobre como determinar los métodos de reducción de la emisión de gases contaminantes.

Sin embargo, los 15 países miembros de la Unión Europea tratarón de lograr en La Haya que el protocolo entrara en vigencia en el 2002, para lograr que las metas pudieran cumplirse, en tal sentido, fue necesario que 55 países ratificarán el documento de Kioto, entre ellos algunas de las economías más industrializadas, aparte de la Unión Europea.

Estados Unidos, Japón, Canadá, Australia y Nueva Zelanda esperaban que el acuerdo final en La Haya no impusiera porcentajes de reducción elevados, que pudieran perjudicar el desarrollo de su industria.

El 25 de noviembre de 2000, tras una prórroga de dos días, se clausuró la Conferencia de la Haya sobre el cambio climático con un rotundo fracaso. No se llegó a acuerdos en la forma de calcular la contribución de los bosques a la absorción del dióxido de carbono de la atmósfera ya que los bosques absorben parte del dióxido de carbono, y el proceso es muy complejo y es difícil de establecer una fórmula matemática que permita evaluarlo y no se acordó tampoco sobre los mecanismos de reducción en la emisión de gases que generan el efecto invernadero. Los países industrializados pusieron trabas a que la reducción de emisiones comience en su propia casa. Los 180 países participantes acordaron suspender la cumbre y volver a reunirse el año siguiente sin haber llegado a un acuerdo sobre algunos de los puntos más importantes, se vuelven a reunir en Julio del 2001 en Bonn (Alemania)

\section{H.- CUMBRE DE MARRAKECH (MARRUECOS)}

Se llevó a cabo entre el 29 de octubre y el 09 de noviembre del 2001 esta fue la IV sesión de la Conferencia de las Partes de la Convención Marco de las Naciones Unidas Sobre el Cam- 
bio Climático (COP7) su objetivo: Traducir a texto jurídico el acuerdo político conseguido en Bonn para salvar los problemas técnicos relacionados con la financiación, el régimen de cumplimiento del protocolo y la normativa de los mecanismos de flexibilidad.

La tarea central de la Conferencia de Marrakech era asegurar la entrada en vigor y la aplicación del Protocolo antes de la Conferencia de Río en 2002; la negociación seguia y en 4 años de reuniones los resultados demuestrarón que el mundo aún no aprende que la Tierra es el único planeta que tenemos.

El acuerdo preveé una reducción de las emisiones de seis gases causantes del efecto invernadero del 5,2 por ciento para los países industrializados en el periodo 2008-2012 con respecto a los niveles de 1990.

Uno de los principales puntos de fricción fue el tema de las áreas verdes que absorben cantidades de CO2 que cada país podría descontar de su cuota obligatoria.

\section{I.- CUMBRE DE NUEVA DELHI. (INDIA)}

La Cumbre de Nueva Delhi (India) fue realizada entre el 23 de octubre y el 01 de Noviembre de 2002, esta fue la VIII sesión de la Conferencia de las Partes de la Convención Marco de las Naciones Unidas Sobre el Cambio Climático (COP8).El tema principal que trataron los países asistentes fue la inminente entrada en vigor del Protocolo de Kioto y la definición del papel de los países en vías de desarrollo en la estrategia mundial para hacer frente al cambio climático, así como tomar medidas para reducir sus efectos. También se anunció que a partir de 2003 se podriá empezar a desarrollar el Ilamado "Mecanismo de Desarrollo Limpio", que permite a los países con obligación de reducción de emisiones que financien medidas de protección del clima en otros países en vías de desarrollo.

La Declaración final de Delhi habló de la necesidad de tomar medidas para luchar contra los efectos del cambio climático y reconoce, como se recoge en los últimos informes del Panel Intergubernamental sobre Cambio Climático (IPCC), la responsabilidad de la actividad humana en el calentamiento global.

Se clausuró la Cumbre con una conclusión definitiva: fortalecer la colaboración internacional sobre cambio climático.

\section{J.- LA CUMBRE DE MILÁN. (ITALIA)}

Fue desarrollada entre el 01 y el 12 de Diciembre del 2003, esta fue la IX sesión de la Conferencia de las partes sobre el Cambio Climático (COP9). La Conferencia parte con la idea de que existen pocas expectativas de avanzar en el cumplimiento de las metas de la reducción 
de emisiones, tras la negativa de Rusia a ratificar el protocolo de Kioto.

Pero la COP de Milán a la que asistieron 166 de los 186 países firmantes de la Convención Marco sobre Cambio Climático de la ONU dio mucho más de sí. En la cumbre se adoptaron decisiones que suponen nuevos pasos en el avance hacia la entrada en vigor del protocolo. Una de ellas tuvo que ver con la puesta en marcha del denominado mecanismo de desarrollo limpio (MDL), un mecanismo previsto en el protocolo que impulsa la transferencia de tecnología en pro del desarrollo y cuyo órgano de gobierno determina, en última instancia, qué proyectos pueden o no ser calificados como MDL y, por lo tanto, pueden acceder o no a los créditos previstos para su financiación. En la anterior COP, celebrada en Bonn, se discutieron qué requisitos debían reunir muchos de esos proyectos pero una de las cuestiones que quedaron pendientes fue aprobar qué criterios y condiciones tenían que cumplir los proyectos de sumideros para poder ser proyectos aceptables por el órgano ejecutivo del MDL.

En la COP de Milán se aprobaron definitivamente esos criterios y condiciones, lo cual significó que el MDL, uno de los mecanismos del Protocolo de Kioto, es ya plenamente operativo. En torno a los proyectos sumidero, además, se produjo uno de los debates más singulares que desembocó en otra decisión relevante. La cuestión se refería a los organismos genéticamente modificados $y$, concretamente, no que una reforestación con especies modificadas genéticamente podía ser aceptada o no como proyecto de mecanismo de desarrollo limpio.

Los detractores de los organismos genéticamente modificados estaban en contra de exportar ese modelo de desarrollo, por lo que hubo algunos países que querían poner como condición el que no se aceptase ningún proyecto basado en especies genéticamente modificadas. Tras un intenso debate se adoptaron dos decisiones: en primer lugar, que esos proyectos debían respetar las exigencias del Convenio de Biodiversidad y el Protocolo de Cartagena y, en segundo lugar, que el prohibir o no ese tipo de proyectos será decisión del país receptor del proyecto

\section{K.-CUMBRE DE BUENOS AIRES. (ARGENTINA)}

Se llevó a cabo entre el 06 y el 17 de Diciembre del 2004 fue la X de la Conferencia de las Partes de la Convención Marco de las Naciones Unidas sobre el Cambio Climático (COP10). El objetivo de la cumbre fue preparar la entrada en vigor del protocolo de Kioto a falta de la adhesión de EE.UU.

\section{L.- CUMBRE DE MONTREAL. (CANADÁ)}

Fue desarrollada del 28 de Noviembre al 09 de Diciembre del 2005 esta fue la XI Conferen- 
cia Sobre Cambio Climático de las Naciones Unidas (COP11). El acuerdo fue mantener las negociaciones que comprometen en principio a los países que han ratificado Kioto, lo que no influye ni a los EE.UU, ni a otros países con grandes emisiones, como China o India.

No se debe confundir la cumbre de Montreal con el protocolo de Montreal que es lo relativo a las sustancias que agotan el ozono, es un tratado internacional diseñado para proteger la capa de ozono reduciendo la protección y el consumo de numerosas sustancias que se ha estudiado que reaccionan con el ozono y se creen que son responsables por agotamiento de la capa de ozono; el Acuerdo fue negociado en 1987 y entró en vigor el $1^{\circ}$ de Enero de 1989. La primera reunión de las partes se celebró en HELSINKI en mayo de ese mismo año. Desde ese momento, el documento ha sido revisado en varias ocasiones, en 1990 (Londres), en 1991 (Nairobi), en 1992 (Copenhague), en 1993 (Bangkok), en 1,995 (Viena), en 1997 (Montreal), y en 1,999 (Beijing). Se cree que si todos los países cumplen con los objetivos propuestos dentro del tratado la capa de ozono podría haberse recuperado para el año 2050.

\section{M.- CUMBRE DE NAIROBI (KENYA)}

Del 06 al 17 de Noviembre de 2006 esta fue la XII Conferencia de las partes de la Convención Marco de las Naciones Unidas sobre el Cambio Climático (COP12), Se aprobó una nueva revisión del protocolo de Kioto en el 2008 y la ayuda a los países pobres a adaptarse al Cambio Climático. Otra idea es la estabilizar las concentraciones de $\mathrm{CO} 2$, en la atmósfera reduciendo las emisiones en $50 \%$ sobre los valores del año 2000, Ilegando a estabilizarse para el año 2050.

\section{N.- CUMBRE DE BALI (INDONESIA)}

Se llevó a cabo del 03 al 14 de Diciembre del 2007 fue la XIII de las Partes de Convención Marco de las Naciones Unidas sobre el Cambio Climático (COP13), tuvo como finalidad tomar acciones internacionales frente al calentamiento del planeta, así como negociar un nuevo tratado que sustituya al de Kioto.

\section{O.- CUMBRE DE POZNAN (POLONIA)}

Se desarrolló entre el 01 y el 12 de Diciembre del 2008, fue la XIV sesión de la Conferencia de las partes de la Convención Marco de las Naciones Unidas sobre el Cambio Climático 
(COP14). Se acordó el impulsar las ayudas destinadas a los países pobres para hacer frente a los efectos del Cambio Climático. Mantienen la referencia de Bali sobre la reducción de emisiones de GEI entre un $25 \%$ y un $40 \%$ para los países industrializados.

\section{P.- CUMBRE DE COPENHAGUE. (DINAMARCA)}

La XV Conferencia Internacional sobre el Cambio Climático (COP15), organizada por las Naciones Unidas, se celebró en Copenhague (Dinamarca), del 07 al 18 de Diciembre del 2009. Allí se elaborarán los objetivos para reemplazar los del protocolo de Kioto, que terminó en 2012.

El objetivo de la conferencia, según los organizadores fue "la conclusión de un acuerdo jurídicamente vinculante sobre el clima, válido en todo el mundo, que se aplique a partir de 2012."

Por otra parte, se propuso como objetivo para la reunión de Copenhague, establecer fuertes compromisos vinculantes de emisiones para los países desarrollados ricos de al menos $40 \%$ por debajo de los niveles de 1990 para apoyar a los países más afectados por el cambio climático, objetivo que no se cumplió.

En dicha cumbre debió existir un acuerdo que garantizace que las emisiones responsables del cambio climático se reduzcan lo suficiente y asegurar así que el aumento de la temperatura global se mantenga por debajo de los $+2^{\circ} \mathrm{C}$, sin embargo esto no paso.

\section{Q.- CUMBRE DE CANCÚN. (MEXICO)}

La XVI Conferencia de la Organización de Naciones Unidas sobre el Cambio Climático (COP16) se celebró en Cancún del 29 de noviembre al 10 de diciembre del 2010, donde más de 190 países del mundo debieron fijar sus posiciones sobre la reducción de la emisión de gases de efecto invernadero, así como realizar una ronda de negociaciones para sustituir o prorrogar el protocolo de Kioto para la protección del Medio Ambiente. Se acuerda en esta reunion un Fondo Verde, monto que servira para ayudar a los paises en desarrollo para que puediesen adaptarse a las consecuencias del cambio climatico.

\section{R.- CUMBRE DE DURBAN. (SUDAFRICA)}

El protocolo de Kioto vencido el 2012, su necesaria continuación se empezó a gestar en las cumbre de Bali 2007 (cop13), y de Copenhague 2009 (COP15). Pero tras el fracaso de la COP15, la COP16 de Cancún (2010) se restableció la ruta hacía la renovación de Kioto en 
la cumbre de Durban.

Durban es un hito clave para el protocolo de Kioto, ya que la falta de acuerdo sobre un segundo período de compromiso se traducirá en que el protocolo caiga en la inercia de facto: existen, pero carece de propósito central. Mientras tanto. Los negociadores lograron llegar a un acuerdo para tener en el 2015 un "instrumento legal vinculante", que limite las emisiones de los países a partir del año 2020. Pero Estados Unidos sigue fuera del Protocolo, y lo acompañan Rusia, Canadá y Japón ya que decidierón no formar parte del segundo periodo de compromisos de Kioto, que iniciaría en enero del 2013 y que no tiene aún fecha de término (2017 o 2020). Al final, en el segundo periodo, Kioto solo controlará el 15\% de las emisiones. El resto de países se mantendrá con los compromisos voluntarios adoptados en Cancún, que según la ONU, no alcanzan para estabilizar el calentamiento de la Tierra.

\section{S.- CUMBRE DE DOHA. (QATAR)}

Se celebro desde el 26 de noviembre al 7 de diciembre del año 2012, la 18 ${ }^{\text {a }}$ Conferencia de las Partes (COP) y la $8^{\text {a }}$ Conferencia de las Partes en calidad de Reunión de las Partes en el Protocolo de Kyoto (CMP 8) en el Centro Nacional de Convenciones de Qatar en Doha.

Las Partes adoptaran finalmente un paquete de decisiones Ilamado el Portal Climático de Doha. Dos de los logros más importantes del Portal Climático de Doha son la adopción formal del segundo período de compromiso del Protocolo de Kyoto para cubrir los 8 años, desde el 1 de enero de 2013 al 31 de diciembre de 2020, y continuar con el impulso hacia un nuevo acuerdo legalmente vinculante para 2020.

Algunos resultados del Portal Climático de Doha fueron los siguientes:

- Con respecto a la visión común, los países confirmaron su acuerdo de limitar el aumento de la temperatura mundial global por debajo de 2 grados hacia 2050, y para que el nivel más alto de emisiones ocurra lo antes posible conforme a los últimos avances de la ciencia, sobre los principios de equidad, responsabilidad común pero diferenciada, y ofreciendo apoyo a los países en desarrollo.

- Los países desarrollados que participan en el segundo período de compromiso del Protocolo de Kyoto (Partes del Anexo I) se han comprometido a reducir sus emisiones globales en al menos 18\% por debajo de los niveles de 1990 durante el período 2013-2020. Estos países deben 'reconsiderar' sus objetivos de reducción de emisiones para el segundo período de compromisos en abril de 2014, y pueden aumentar sus objetivos conforme a emisiones globales reducidas en un 25-40\% por debajo de los niveles de 1990 hacia 2020 (el nivel necesario para limitar la temperatura mundial al límite acordado de 2 grados) 


\section{T.- CUMBRE DE VARSOVIA. (POLONIA)}

Esta Conferencia de Cambio climático se realizó en Varsovia Polonia, celebrada del 11 al 22 de noviembre del 2013 conocida como COP 19 con el propósito de conocer los avances en forma detallada, de las negociaciones especiales, sobre el cambio climático. Se pudo apreciar el poco interés que tienen los grandes países industrializados en contribuir a la reducción de emisión de gases.

Durante las últimas conferencias de las Naciones Unidas (ONU) sobre cambios climáticos se ha visto la irresponsabilidad de los países industrializados, y los países en vías de desarrollo como ser los Estados Unidos, Polonia, China, India, Rusia, Australia, Japón, Canadá y Unión Europea, simulan actuar implementando medidas de reducir y detener el calentamiento global del Cambio Climático, minimizando el apoyo técnico y financiero en cumplimiento de los objetivos de los procesos del cambio climático como medidas de mitigación, adaptación y otros.

En esta Conferencia de Cambio Climático, se establecen acuerdos sobre emisiones y mecanismos sobre perdidas y daños. Situación que ha sido denunciada por la UNESCO causando grandes daños a la economía, al medio ambiente y la salud humana, debido a la falta de capacidad, para tratar el dióxido de carbono, emitido en la atmósfera. Por lo que se debería de tomar algunas medidas sobre las grandes industrias de carbón tradicionales, debiendo utilizarse en otras fuentes para generar energía limpias, y en relación a las plantas se debe implementar nuevas tecnologías que permitan atrapar las emisiones antes de que contaminen la atmosfera. Y así lograr una reducción de la emisión de dióxido de carbono Según el Secretario de Naciones Unidas (ONU) BanKi-Moon, es de gran preocupación el tema del cambio climático a nivel mundial, manifestando que "el calentamiento global amenaza las presentes y futuras generaciones, en virtud del aumento de emisiones de gases de efecto invernadero". El principal objetivo de esta conferencia fue lograr un acuerdo para el año 2015 en referencia a la reducción de emisiones de gases contaminantes, sin embargo, a este acuerdo se oponen varios países, entre ellos el anfitrión de esta conferencia, Polonia, ya que su modelo económico se basa en el carbón.

\section{U.- CUMBRE DEL CLIMA NUEVA YORK. (U.S.A)}

120 líderes mundiales se reunieron el 23 de Septiembre en la ciudad de Nueva York, para celebrar la Cumbre del Clima 2014 organizada por la ONU. El objetivo: advertir sobre la delicada situación de nuestro Planeta y exigir compromisos reales a las diferentes naciones contra el cambio climático.

El Secretario General de la ONU Ban Ki-moon inauguró esta importante cita internacional, 
con un mensaje claro: " Debemos orientar el mundo en una nueva dirección, el cambio de clima es la cuestión definitoria de nuestra época. Nuestra respuesta definirá nuestro futuro". El Presidente de Estados Unidos Barack Obama fue otro de los grandes protagonistas recordemos que EEUU es el segundo país con más emisiones de efecto invernadero-. Su mensaje: "El cambio climático es la amenaza que más define a este siglo".

El principal objetivo de esta cumbre celebrada en Nueva York fue que los países pongan sobre la mesa sus compromisos para la protección del medio ambiente y queden reflejados en la negociación de un nuevo tratado sobre el cambio climático a celebrarse a finales de este año 2014 en Lima, Perú y 12 meses después en la Cumbre de París será donde se cerrará la negociación.

¿Qué se ha hecho hasta ahora? Los líderes mundiales han prometido 5.000 millones de dólares a favor de la sostenibilidad, una inversión que puede ayudar a acabar con la combustión de carbón, petróleo y gas, y apostar por fuentes de energía renovables. Otro gran objetivo que busca la cumbre es acabar con la destrucción de los bosques. Estados Unidos, Canadá y la Unión Europea firmaron una declaración para eliminar la deforestación completamente para el 2030. Por otra parte, el sector financiero se acaba de comprometer a aportar 200.000 miIlones de dólares para mitigar el calentamiento global.

Según la comunidad científica," La situación de nuestro Planeta es grave". Si para 2020 no entra en vigor una convención con nuevos recortes de emisiones, será demasiado tarde para contener el calentamiento global por debajo de los niveles considerados peligrosos. Las evidencias científicas existen: los casquetes polares se derriten más rápido que nunca y la subida del nivel del mar desde 1992 fue el doble que en todo el siglo pasado.

Por otra parte, el aumento de las temperaturas origina sequías que afectan gravemente a las producciones agrícolas y por ende, a muchas naciones del mundo cuya economía depende de ello. (Twenergy, 2014)

\section{V.- CUMBRE DE LIMA. (PERU)}

La Cumbre Climática COP20 se realizará en diciembre 2014 en Lima, Perú y será determinante para sentar las bases de lo que será un acuerdo global de compromisos para reducir las emisiones de gases de efecto invernadero y frenar el calentamiento del planeta, tiene como objetivo tener el borrador de un acuerdo global.Ese borrador tendrá que discutirse en la siguiente cumbre del 2015, a desarrollarse en París, Francia.

Si bien la COP20 será este próximo diciembre, el proceso incluye una serie de negociaciones previas para avanzar en tener un documento final. Los avances que se han logrado a la fecha son:

1) Fondo verde. Se aprobó los mecanismos que faltaban definir para que quede operativo el 
fondo verde del clima. Con esto, se podrán recibir los aportes económicos de las economías desarrolladas para que los países en vías de desarrollo, puedan hacer frente a los impactos negativos del cambio climático.

2) Compromisos de reducciones. La Unión Europea propuso la reducción de sus emisiones al 2030 y al 2050. A esto se suma que Estados Unidos anunció una serie de medidas para reducir sus emisiones de plantas de generación de energía en base a carbono, como parte de su plan de acción climático. Además existe posibilidad de que China ponga sobre la mesa su compromiso de reducción. Estas economías son las que más emisiones registran en el mundo. 3) Contribuciones. En el Grupo de Trabajo Ad Hoc para la plataforma de Durban se lanzó un primer borrador de lo que será la información de las contribuciones nacionales que cada país pondrá sobre la mesa el primer trimestre del 2015. Las contribuciones nacionales son los compromisos de cada país sobre cómo reducirá sus emisiones en base a su propia realidad.

Estos son avances importantes para lograr una negociación efectiva en la COP20 y se tenga un documento que reemplace al Protocolo de Kyoto. (Ortiz, 2014)

\section{W.- CUMBRE DE PARIS (FRANCIA).}

Francia fue designada país anfitrión de la XXI Conferencia de las Partes (COP21) de la Convención Marco de las Naciones Unidas sobre el Cambio Climático (CMNUCC) con el principal objetivo de llegar a un nuevo acuerdo internacional sobre el clima previsto para después de 2020.

Los retos de la COP 21 será constituir una etapa decisiva en la negociación del futuro acuerdo internacional previsto para después de 2020, con el objetivo de que todos los países, y entre ellos los mayores emisores de gases de efecto invernadero países desarrollados y países en desarrollo estén vinculados por un acuerdo universal sobre el clima.

Francia desea un acuerdo ambicioso, aplicable a todos, con el fin de alcanzar el objetivo de limitar a dos grados el calentamiento global del planeta.

El acuerdo deberá traducir un cambio de orientación que tenga en cuenta el reto climático no ya como una necesaria "carga compartida" en lo que se refiere a las emisiones, sino como una oportunidad para crear empleo y riqueza a través del desarrollo de nuevos modelos de producción y de consumo.

Para que dicho acuerdo entre en vigor en 2020, al finalizar el segundo periodo de compromiso del Protocolo de Kioto, las siguientes COP rematarán los detalles del acuerdo. La presidencia de la COP tiene un papel esencial no sólo durante la COP, que es en realidad una serie de reuniones simultáneas durante dos semanas, sino también a través de la organización de varias reuniones preparatorias a la COP.

Francia tiene un papel activo y sus emisiones de gas con efecto invernadero por habitante son 
ya de las más bajas en los países desarrollados. Además, Francia prepara su propia transición energética hacia un futuro de poco carbono. Francia Junto a sus socios de la Unión Europea, hará todo lo posible para que la Unión conserve su puesto puntero en la lucha contra el cambio climático. El presidente en su discurso de apertura de la conferencia medioambiental, expresó que Francia defenderá una posición ambiciosa, centrada en el objetivo de reducir en $40 \%$ las emisiones de gases con efecto invernadero en 2030 y $60 \%$ en 2040 (respecto a 1990), en el marco de las próximas discusiones europeas. Por último, Francia se comprometerá a construir durante el quinquenio una auténtica diplomacia medioambiental.

\section{CONCLUSIONES}

1.- Es de gran preocupación de los países del mundo, los problemas existentes del medio ambiente sobre todo en relación a la variabilidad del clima, y es donde aúnan esfuerzos para la creación de un instrumento que regule las condiciones que generan las industrias con los procesos de contaminación y que degradan el medio ambiente afectando el cambio climático como ser: la emisión de gases, fabricación de productos químicos, refrigerantes, aerosoles y otros contaminantes peligrosos.

2.- Para lograr contrarrestar los problemas del cambio climático los países de las Naciones Unidas, firmaron el protocolo de Kioto, con el fin de reducir la emisión de gases de efecto invernadero que afecta los ecosistemas de la atmosfera, agua, y suelo en perjuicio de un mejor ambiente y una mejor calidad de vida humana. Por lo que es una necesidad mundial mantener un equilibrio entre el uso de los recursos naturales y el crecimiento económico de los países desarrollados y en vía de desarrollo, manteniendo un desarrollo sostenible de los recursos naturales y materiales.

3.- Con el Convenio de Montreal se regula la protección de la Capa de Ozono que es de interés mundial y para su aplicación se crea el Protocolo de Montreal donde obliga a los países miembros a cumplir para lograr el bienestar de la población mediante medidas o compromisos de reducir la emisión de gases y otros factores como: La deforestación, contaminación atmosférica y pérdida de diversidad y uso de productos químicos entre otros, que perjudican la Capa de Ozono.

4.-El agotamiento de la capa de ozono como producto de la actividad industrial y del uso de ciertas sustancias en diferentes actividades comerciales y domésticas, es el primer gran problema ambiental que la humanidad ha tenido que reconocer como la consecuencia de un particular desarrollo tecnológico y económico de la sociedad actual. Los acuerdos internacio- 
nales para tratar de recuperar y proteger la capa de ozono Convenio de Viena y Protocolo de Montreal deben ser vistos como el triunfo de la diplomacia internacional, al lograr conciliar los intereses de productores, industriales, países desarrollados, países en vías de desarrollo y de los consumidores en general.

\section{RECOMENDACIONES}

1.- Los países miembros de las Naciones Unidas deben asumir con responsabilidad la protección del planeta tierra mediante los principios generales del ambiente: como el desarrollo sostenible, tecnologías amigables al ambiente, protección y conservación de la diversidad biológica.

2.- Los países desarrollados deben ratificar los convenios del cambio climático especialmente de Protocolo de Kioto, Protocolo de Montreal, Convenio de la Estocolmo y otros instrumentos ambientales internacionales que contribuyen a mantener un medio ambiente saludable en beneficio de todos los seres vivos, facilitando el apoyo técnico y financiero a los países subdesarrollados mediante los diversos programas encaminados al mejor uso de los recursos y la protección y conservación de los mismos.

3.- También los países miembros deben luchar por implementar medidas para reducir el calentamiento global y la desertificación y mitigar los efectos de las sequías y las inundaciones adoptando medidas tales como una mejor utilización de la información pronósticos meteorológicos, las prácticas agrícolas y la conservación de los ecosistemas con el fin de invertir las tendencias actuales y reducir al mínimo la degradación de la tierra y los recursos hídricos, incluso proporcionando recursos financieros en cantidades suficientes y previsibles para aplicar la Convención de las Naciones Unidas de lucha contra la desertificación y el calentamiento global.

\section{BIBLIOGRAFIA}

Alongo, B. (2010). Coordinadora Institutcional, Centro de Integracion y Cooperacion Internacional. CICODI. Obtenido de http://www.upv.es/

Climatico, G. I. (1995). Panel on Climate Change intergovemmental IPCC. Ginebra.

Desarrollo, C. M. (1987). Nuestro Futuro Comun. Oxford University Press.

Monique Bardut, (1991) Directora Ejecutiva y Presidenta Fondo para el Medio Ambiente Organización de las Naciones Unidas y la Ciencia y la Cultura.- Paris.

ONU. (1991). La Ciencia y la Cultura. New York. 
Ortiz, M. (19 jueves de junio de 2014). El Comercio. Obtenido de http://elcomercio.pe/economia/peru/cumbre-cop20-cual-avance-negociaciones-climaticas-noticia-1737170 Secretaria del Ambiente de Paraguay (nov 2012).Protejamos la Capa de Ozono.6 Noviembre . Sánchez, Jorge. (2006) Contexto General. En: UNIDAD TÉCNICA OZONO. Implementación del Protocolo de Montreal en Colombia. Bogotá: La Unidad Técnica Ozono, p. 10-19. wenergy. (martes 25 de Septiembre de 2014). Twenergy. Obtenido de http://twenergy.com/a/ cumbre-del-clima-nueva

VEGA, Miriam. (2006) Tratados internacionales para la protección a la capa de ozono [on line]. s.l. : Programa de las Naciones Unidas para El Medio Ambiente, Agosto. [Citado febrero 2007]. Disponible en http://www.pnuma.org/ozono/tratados.php www. Alianza geográfica Org. /lección Calentaglobal. Derechos reservados Lliam Bird y José Molinelly 